\title{
La typologie des entassements en français
}

\author{
Kahane, Sylvain \& Pietrandrea, Paola \\ Université Paris Ouest \& Modyco CNRS, Università Roma Tre \& Lattice CNRS \\ sylvain@kahane.fr, paolapietrandrea@,gmail.com
}

\section{Introduction}

Nous appelons entassements des constructions caractérisées par le fait que plusieurs éléments viennent occuper une même position régie. Il peut s'agir de coordinations ${ }^{1}$ :

(1) des jeunes \{ cagoulés |^et armés $\}$ ont \{ pillé des magasins $\mid$ brûlé $\{$ des pneus $\mid \wedge$ et des voitures \} \} // (M206)

mais aussi de divers autres phénomènes que nous allons présenter, à commencer par des disfluences et des reformulations comme :

(2) et je voulais pas aller à Addis Abeba // puisque $\{\{\{$ les $\mid$ les $\mid$ les $\mid$ les $\} \mathbf{c} \sim \mid$ les capitales $\} \mid$ les grandes villes $\}$ ne me disaient rien du tout // (D204)

Blanche-Benveniste est probablement la première linguiste à avoir mesuré l'importance de ces constructions dans les productions orales spontanées et avoir proposé de les regrouper en considérant qu'elles relevaient d'un même procédé de listage (Blanche-Benveniste, 1997, Blanche-Benveniste, 1990). Tesnière a pour sa part caractérisé la coordination comme un procédé orthogonal à la dépendance proprement dite (ce que Blanche-Benveniste appelle la rection) et a nommé ce procédé la jonction :

«La jonction consiste à ajouter entre eux des nœuds de même nature. [...] Deux nœuds ne pouvant être jonctés qu'à condition d'être de même nature, et par conséquent d'appartenir au même étage structural, le nœud de jonction devra forcément être horizontal. » (Tesnière, 1959 : 323-326)

Dans les représentations qu'il propose, les dépendances sont verticales, tandis que la jonction est représentée par des traits horizontaux. Voici ce que serait l'analyse de (1) par Tesnière :

$\left(1^{\prime}\right)$

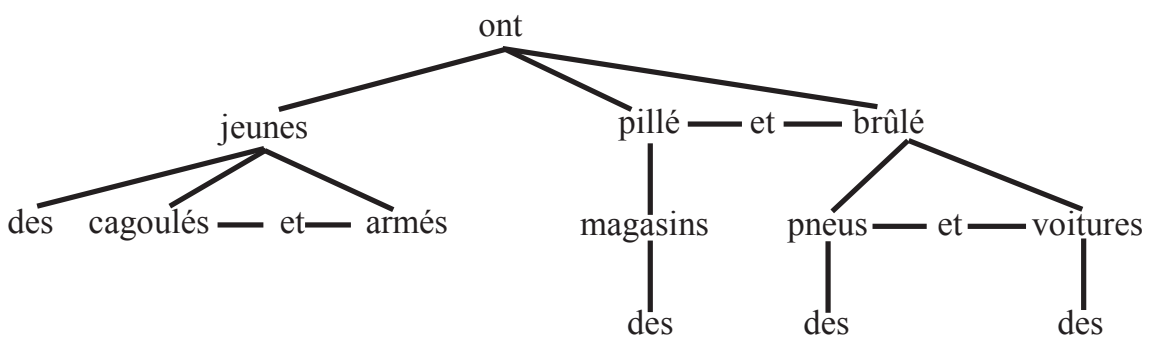

Blanche-Benveniste utilise un procédé similaire (mais inversé) pour la présentation des productions orales en plaçant horizontalement le défilement syntagmatique de l'énoncé et verticalement les listes paradigmatiques d'éléments venant occuper une même position : c'est ce que l'on appelle depuis lors l'analyse en grille (Blanche-Benveniste, 1990, Blanche-Benveniste, 1997), dont nous avons un exemple ci-dessous. (cf. Kahane, à par. pour une discussion plus approfondie) : 
Dans cet article, nous allons nous intéresser à la typologie des entassements. Les travaux de BlancheBenveniste (1990, 1995), Bilger (1999), Guénot (2006) ont montré que les entassements ont des fonctions sémantiques et pragmatiques variées : ils peuvent résulter de disfluences, mais ils peuvent surtout servir à établir des relations entre dénotations, à créer de nouvelles dénotations, à reformuler, à exemplifier, à préciser, à intensifier. Dans cet article, nous proposons une classification hiérarchique, fonctionnelle et formelle à la fois, des entassements.

Il est à noter que si les coordinations font l'objet d'une abondante littérature et sont considérées dans toutes les tentatives de modélisation des langues, aucun modèle formel à notre connaissance (même limité à un niveau syntaxique) ne prend en compte la notion d'entassement dans toute sa généralité et que la plupart des modèles formels ne considèrent pas les reformulations. On peut peut-être trouver une explication à cela dans le fait que si la coordination a toujours été considérée comme relevant de la compétence des locuteurs, la reformulation a été longtemps considérée comme un problème dû à la performance. Nous verrons que, même pour la coordination, les études restent partielles. Nous montrerons en effet que l'on peut distinguer deux grands types de coordinations, que nous appellerons respectivement relationnelles et non relationnelles, et que seules les premières sont généralement considérées dans la littérature, alors que les deuxièmes constituent plus de la moitié des occurrences de coordinations dans certains corpus.

Nous commencerons notre étude par une caractérisation plus précise de l'entassement et par une description formelle de la structure des entassements. Puis, nous présenterons la typologie proprement dite. Nos données sont extraites du corpus annoté dans le cadre du projet Rhapsodie, un corpus de 3 heures, soit 36000 mots, de français oral spontané, pour lequel les entassements ont été systématiquement annotés (Benzitoun et al., 2009, 2010) ${ }^{2}$. Les numéros à la suite de nos exemples, comme D005 ou M206 (D pour dialogue, M pour monologue), indiquent l'échantillon dont l'exemple est extrait. L'annotation des types d'entassements est en cours et cet article peut être vu comme une présentation des types retenus pour cette annotation.

Nous proposons de classifier l'ensemble des entassements sous deux grandes catégories, coordinations et entassements de dicto :

- les coordinations ou entassements de re construisent une expression dont la dénotation diffère de la dénotation des conjoints; autrement dit, la coordination est une construction (et donc un signe linguistique) qui possède une contribution sémantique réelle ;

- les entassements de dicto établissent des relations entre formulations ; soit les conjoints n'ont pas réellement de dénotation (disfluence), soit les conjoints dénotent la même chose de différentes façons ; l'entassement de dicto n'a donc pas de contribution sémantique et il n'est pas un signe linguistique, mais une configuration structurale.

Chacun des deux groupes inclut un certain nombre de sous-types, qui sont identifiés sur la base d'un certain nombre de critères formels et fonctionnels.

\section{Définition et structure des entassements}

Avant de définir l'entassement proprement dit, il faut caractériser la rection. Nous introduirons également les notions d'unité rectionnelle et d'unité illocutoire, que nous utiliserons dans notre typologie. 


\subsection{Rection, unité rectionnelle et unité illocutoire}

Nous appelons rection ${ }^{3}$ la propriété qu'ont les mots d'imposer des contraintes à d'autres mots : il s'agit de contrainte sur 1) la nature ; 2) les marqueurs morphologiques et syntaxiques, 3) la place. Ainsi un verbe impose-t-il à son complément d'objet, 1) d'être un groupe nominal ou bien 2) d'être à l'accusatif s'il s'agit d'un pronom ou d'être translaté par l'infinitif ou le complémenteur que s'il s'agit d'un verbe ; 3) d'être placé après lui s'il s'agit d'un groupe nominal. Nous appelons unité rectionnelle (UR) les segments maximaux pour la rection.

En plus de la segmentation en UR du texte, nous considérons une segmentation en unités illocutoires (UI). Une UI correspond à un acte illocutoire (une assertion, une question, une injonction). Elle peut être composée de plusieurs composantes illocutoires (chacune correspondant à une UR). La composante qui porte la force illocutoire est le noyau (c'est une vague incroyable en (3); il m'a été commandé en (4)). Les autres composantes sont appelées des adnoyaux (pré- ou post-noyaux selon leur position par rapport au noyau) (Benzitoun et al., 2010, Deulofeu et al., 2010). Nous notons // la frontière entre $\operatorname{deux}$ UI et $<$ et $>$ la limite entre le noyau et les adnoyaux qui le précèdent ou le suivent et nous notons entre "..." des éléments qui portent une certaine force illocutoire sans être pour autant totalement indépendant et que, dans Kahane \& Pietrandrea (2012), nous avons appelé des UI associées :

(3) et alors < la critique déferlante < c'est une vague incroyable // (D210)

(4) il m'a été commandé > ce livre "attention" "hein" // (D210)

Il n'est pas rare qu'une UR se prolonge au delà d'une UI. Nous notons //+ une frontière d'UI qui n'est pas une frontière d'UR :

(5) $\$$ L1 alors $<$ qui vous regarde //

\$L2 c'est un Chinois //+ très riche // (D210)

Un tel cas, appelé traditionnellement épexégèse (Bally, 1932), se repère à la prosodie et à l'interprétation : il y a bien deux assertions ici : 'c'est un Chinois qui me regarde' et 'ce Chinois est très riche', comme le prouve le fait que la deuxième assertion peut être modifiée par des adverbes d'énonciations (comme franchement, malheureusement ou heureusement) qui prennent dans leur portée sémantique des illocutions :

(6) $\$$ L1 alors $<$ qui vous regarde //

\$L2 c'est un Chinois //+ heureusement $<$ très riche //

Il est même possible qu'une UR se prolonge sur deux tours de parole, lorsqu'un locuteur s'appuie l'UI de son interlocuteur pour produire la sienne :

(7) \$L1 donc < moi < "ben" \{ je vais $\mid\{$ je $\mid$ je $\}$ prends le mét $\sim$ je prends le métro $\}$ le matin "bon" jusqu' au Palais Royal //+

\$L2 à quelle heure "excusez-moi" //

\$L1 "oui oui" je prends le métro le matin à huit heures et demie // (D001)

Nous verrons que les entassements, qui par définition se situent à l'intérieur d'une UR, peuvent assez facilement se trouver sur plusieurs UI ou plusieurs tours de parole. C'est même une caractéristique de certains types d'entassements.

\subsection{Entassement, couche, jonctif, clôtureur}

L'entassement est une dimension orthogonale à la rection : on dit qu'un segment Y s'entasse sur un segment $\mathrm{X}$ si $\mathrm{Y}$ vient occuper la même position régie que $\mathrm{X}$. Nous notons $\{\mathrm{X} \mid \mathrm{Y}\}$ le fait que $\mathrm{X}$ et $\mathrm{Y}$ sont 
entassés (cf. (1) et (2) au début de l'article). Dans l'entassement $\{\mathrm{X} \mid \mathrm{Y}\}, \mathrm{X}$ et Y sont chacun une couche de l'entassement. Un entassement peut avoir deux couches ou davantage. Dans une couche, on distingue quatre types d'éléments. Les deux premiers sont les conjoints et les joncteurs ${ }^{4}$.

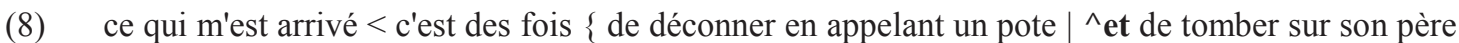
\}$/ /$ mais "euh" "euh" $\left\{\left\{\{\right.\right.$ se $\mid$ se $\mid$ se $\}$ gourer $\mid \wedge$ et ${ }^{\wedge}$ puis chauffer comme ça $\} \mid{ }^{\wedge} \mathbf{c}^{\prime}$ est-à-dire \{ dragouiller la mère $\mid \wedge$ ou draguer $\mid \wedge$ ou faire une déclaration d'amour à la mère $\}\}<$ non // (D207)

Les conjoints sont les syntagmes qui viennent occuper la même position syntaxique régie et qui s'entassent dans cette position :

(9) a. ça m'est arrivé de déconner en appelant un pote

b. ça m'est arrivé de tomber sur son père.

Les joncteurs sont les éléments qui lient les conjoints (nous les marquons en les faisant précéder d'un ^). Les joncteurs occupent un rôle uniquement à l'intérieur de l'entassement. En particulier, si l'on conserve uniquement une couche de l'entassement, les joncteurs ne peuvent pas être maintenus :

(10) a. *ça m'est arrivé et de tomber sur son père

b. *ça m'est jamais arrivé c'est-à-dire de dragouiller la mère.

On trouve également dans les entassements des adverbes, notamment paradigmatisants (Nølke, 1983), comme seulement ou aussi, en gras dans l'exemple suivant :

(11) ^mais c'est aussi \{ une conférence $\{\mathrm{de} \mid \mathrm{d}$ ' $\}$ histoire de l'art $\mid$ une conférence d'esthétique $\}$ parce que [ comme vous verrez $<$ j'ai une approche $\{$ de $\mid$ de $\}$ l'art $\{$ qui est $\{$ assez $\mid$ assez $\}$ généraliste $\left.\right|^{\wedge}$ et qui "euh" mêle $\{\{$ plusieurs $\mid$ "euh" plusieurs $\}$ points de vue $\mid \wedge$ et donc $\{$ pas seulement le point de vue de la philosophie $\mid \wedge$ mais aussi $\{$ celui de l'histoire $\mid$ celui de la sociologie $\mid$ et caetera | et caetera \} \} \} \} ] // (M202)

A la différence des joncteurs, ceux-ci peuvent être maintenus si une seule couche est conservée :

(12) a. mon approche (ne) mêle donc pas seulement le point de vue de la philosophie

b. mon approche mêle aussi le point de vue de l'histoire.

Ils sont donc « visibles » pour la rection, mais occupent une position différente de celle du conjoint : alors que les conjoints ne peuvent être effacés, les adverbes paradigmatiques le peuvent.

Le dernier type d'éléments que l'on trouve dans les entassements ont la propriété de pouvoir clore un entassement, comme et caetera (voir exemple précédent). Nous les appellerons des clôtureurs. Ils ne peuvent occuper que la dernière couche d'un entassement. Le cas de et caetera est exemplaire, puisque celui-ci ne peut apparaître que dans la dernière couche d'un entassement :

(13) *mon approche mêle et caetera.

Mais il existe aussi de nombreux clôtureurs qui sont formés d'un joncteur et d'un type particulier de conjoint, comme tout ça, que nous appelons, à l'instar d'Overstreet (2005), un extenseur ${ }^{5}$ :

(14) et "euh" "bon" "ben" ça pose des problèmes \{ de maintenan | "enfin" de maintenance "euh" | \{ \{ de $\mid$ de \} mise à jour $\left.\right|^{\wedge}$ et tout ça \} "euh" \} // voilà // (D005) 


\subsection{Entassements plus problématiques}

Nous avons présenté la situation standard des entassements, mais plusieurs configurations s'en éloignent. Premièrement, il n'est pas rare qu'un entassement soit discontinu, c'est-à-dire que deux couches successives ne soient pas adjacentes. Nous notons l'entassement discontinu des couches $\mathrm{X}$ et $\mathrm{Y} \operatorname{par}\{\mathrm{X} \mid\}$ $\ldots\{\mid \mathrm{Y}\}$ :

(15) \$L1 puisque finalement $<$ ça fait "euh" \{ quarante-huit ans |\} que vous êtes au Kenya //+ \$L2 \{| quarante-huit ans $\} / /\{$ oui | oui $\} / /(\mathrm{D} 204)$

Mais nous verrons que certains types d'entassements, notamment dans la reformulation, sont facilement discontinus.

Le joncteur $n i$ est particulier, puisqu'il intégre une négation, c'est-à-dire un adverbe paradigmatisant. Il peut résulter de cela qu'il occupe la position d'un tel adverbe (c'est-à-dire d'un pas), plutôt que celle du joncteur ${ }^{6}$ :

(16) parce qu' \{ on remarque "euh" \{ ass $\sim$ "euh" | de façon "euh" $\} \& \mid$ on remarque bien que le salaire n'est ${ }^{\wedge}$ ni en adéquation $\{\{$ avec le nombre d'années \& "euh" | avec le nombre d'études suivies $\}$ |

$\wedge^{\wedge}$ ni avec le travail \{à fournir | personnel que l'on fournit \} \} // (M103)

Il existe enfin des configurations particulières de joncteurs et d'adverbes paradigmatiques qui permettent l'omission du second conjoint (qui serait en fait ici une pure répétition du premier conjoint) :

(17) ils demandent vingt-deux milliards de dollars $\{$ en contrepartie de suppressions d'emplois $\mid \wedge$ mais pas seulement \} // (M206)

\subsection{Coordinations sans entassement}

La conséquence de notre définition de l'entassement est que deux cas de coordinations ne sont pas considérés comme des entassements et ne sont pas étudiés ici. Le premier est la coordination reliant deux ou plusieurs UR :

(18) depuis avant la guerre j'avais rencontré euh Monfred Henri de Monfred qui venait faire des conférences à à Mulhouse // et et et c'est un type euh passionnant // et j'ai toujours lu tous ses livres // et j'avais absolument envie d'aller dans la corne de l'Afrique la l'Éthiopie la Somalie euh l' Ora l' Arabie tout ça // (D204)

En effet, par définition, les UR sont des unités maximales pour la rection et par conséquent, on ne peut pas considérer que deux UR coordonnées occupent une même position régie, puisque position régie, il n'y a pas. On considère également que chacune de ces UR réalise un acte illocutoire et forme donc une UI.

L'autre cas est illustré par des coordinations comme :

(19) on veut bien parler avec vous //+ mais $\{\mathrm{a} \sim$ après $\}$ le déménagement // (D006)

(20) normalement $<$ c'est du bois de hêtre dessous //+ et \{ qui est p \& qui est laqué noir \} // (D009)

où le deuxième conjoint n'occupe pas une même position régie que le premier conjoint. Nous considérons qu'il s'agit ici d'une coordination entre unités illocutoires. Le locuteur fait en fait deux assertions : 'on veut bien parler avec vous' mais 'on veut parler après le déménagement'. Et il réalise cela en produisant une unique UR (on veut bien parler avec vous après le déménagement) en deux UI. On modélise ces 
coordinations sans recours à l'ellipse, grâce à la distinction de deux unités syntaxiques distinctes, UR et UI.

\section{Coordinations}

Nous distinguons deux grands types de coordinations : les coordinations relationnelles, c'est-à-dire les coordinations au sens classique du terme, et les coordinations non relationnelles.

\subsection{Coordinations relationnelles}

Les coordinations relationnelles sont des coordinations où chaque conjoint dénote un élément différent et où l'entassement complet possède une dénotation qui est fonction des dénotations de ses conjoints ${ }^{7}$. Nous distinguons deux grands types de coordinations relationnelles, les coordinations additives et les coordinations alternatives, selon la fonction qui relie la dénotation de l'entassement à celles des conjoints.

\subsubsection{Coordinations additives}

Dans une coordination additive, la dénotation de l'entassement est la réunion des dénotations des conjoints. Les coordinations additives sont généralement marquées par la présence d'un joncteur conjonctif, en premier lieu et.

(21) alors < passons maintenant \{ au détail des mesures discutées $\mid \wedge$ et aux attentes $\{$ des syndicats $\mid$ ^et du patronat \} \}// (M206)

Les coordinations additives peuvent aussi être non marquées par des joncteurs:

(22) un sondage \{ BVA | Les Échos | France Info \} réalisé les \{treize $\mid \wedge$ et quatorze $\}$ février derniers // (M206)

(23) c'est à ceux de votre tradition \{ que nous avons arraché \{ le suffrage universel | la liberté d'association \} | que nous avons arraché la liberté d'association | que nous avons arraché \{ la liberté de la presse | le droit de grève | le droit à l'instruction \} \} // (D206)

En plus du joncteur et, on trouve le joncteur $n i^{8}$ :

(24) on s'est retrouvés sans $\left\{\right.$ eau $\left.\right|^{\wedge}$ ni gaz $\left.\right|^{\wedge}$ ni téléphone $\left.\right|^{\wedge}$ ni électricité $\} / /$ (D003)

(25) car "euh" les formations linguistiques qui sont données aux immigrés qui arrivent ne sont pas faites pour les gens $\left\{\right.$ qui ne parlent pas du tout $\left.\right|^{\wedge}$ ni qui n'ont jamais été à l'école $\} / /$ (D002)

Ainsi que puis et ainsi que:

(26) $\{\mathrm{j} \sim \mid \mathrm{je}\}$ connaissais des gens dans le septième qui mettaient leurs enfants \{ d'abord dans le public | ^puis dans le privé \} pour \{ qu'ils puissent passer après éventuellement \& "euh" "euh" | $\wedge$ et qu'ils soient plus forts $\} / /$ (D002)

(27) nous avons été sous très gros bombardements \{ américains $\left.\right|^{\wedge}$ puis anglais $\} / /$ (D003)

(28) alors < ce que je souhaiterais faire de ma vie < c'est \{ devenir professeur d'italien à savoir certifié | donc "euh" enseigner $\left\{\right.$ au collège $\left.\right|^{\wedge}$ ainsi ^${ }^{\wedge} \mathbf{q u}$ ' au lycée $\}$ \} // (M103) ${ }^{9}$

Le lexème mais possède une valeur contrastive supplémentaire ${ }^{10}$ et se combine souvent avec des négations, mais il est bien additif et commute avec et : 
(29) "euh" mon père était un autodidacte \{ \{ venant d'un milieu très pauvre | ayant fait aucune étude $\left.\right|^{\wedge}$ mais $\{$ ayant une passion $\{$ de la littérature $\mid \wedge$ et du savoir $\} \mid \wedge$ et ayant accumulé un énorme savoir d'autodidacte $\}$ \} // (D205)

(30) je travaille à la préfecture de Paris qui \{ n'est pas connue |^mais néanmoins existe $\}$ "euh" // (D001)

(31) mes souvenirs les plus anciens sont "je crois" des souvenirs \{ de Mulhouse |^mais sûrement pas de Cannes \} // (D204)

Un dernier cas de joncteur additif est comme ${ }^{11}$ :

(32) il y a $\{$ des $\mid$ des $\}$ traditions de famille qui se transmettent $\{$ dans le domaine religieux $\mid \wedge$ comme dans tout le domaine du travail $\} / /$ (D101)

On peut contraster cet emploi de comme avec un emploi régi :

(33) il s'est voulu comme \{ un homme d'idées | un homme de concepts \}// (D205)

Seul l'emploi régi peut être coordonné ${ }^{12}$ :

(34) il s'est voulu comme un homme d'idées $\mid \wedge$ et comme un homme de concepts \}

(35) *elles se transmettent dans le domaine religieux comme dans le domaine du travail et comme dans le domaine de la famille

A la différence de Rousseau (2001), qui a proposé une classification des coordonnants dans un cadre de logique naturelle, nous ne retenons pas donc parmi les joncteurs, car celui-ci ne remplit pas notre condition syntaxique définitoire. Si l'on prend l'exemple (36), on voit que si l'on garde uniquement la deuxième couche de l'entassement, donc peut être maintenu :

(36) ce que je souhaiterais faire de ma vie $<$ c'est donc enseigner au collège

\subsubsection{Coordinations alternatives}

Une coordination alternative marque que les éléments dénotés par les conjoints sont potentiellements substituables les uns aux autres (Mauri, 2008 : 47). Les coordinations alternatives sont marquées par la présence d'un joncteur disjonctif (ou, soit, ou bien).

(37) allez // avec Messi \{ qui va chercher le corner $\left.\right|^{\wedge}$ et qui va trouver \{ le corner $\left.\right|^{\wedge}$ ou la touche | ^ou la sortie de but $\}$ \}// (D203)

Certaines coordinations alternatives sont marquées par la présence d'une conjonction comparative :

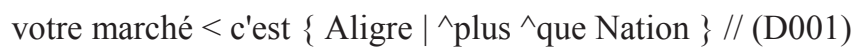

Comme dans les coordinations en ou, les conjoints sont mis en balance, mais avec un asymétrie plus prononcée. Plus encore que pour comme, le statut de joncteur de plus que peut être discuté et une coordination, comme en (a), n'est pas totalement exclue, même si on lui préférera nettement (b) :

(39) a. ?? votre marché < c'est Aligre plus que Nation et plus que Voltaire

b. votre marché $<\mathrm{c}^{\prime}$ est Aligre $\mid \wedge$ plus ${ }^{\wedge}$ que $\{$ Nation $\mid \wedge$ ou Voltaire $\}$ \} 


\subsection{Coordinations non relationnelles}

Nous reconnaissons à l'instar des études conduites par Bonvino et al. (2009), sur l'italien écrit, l'existence en français oral, de nombreux types de coordinations non relationnelles : il s'agit de coordinations qui ne posent pas une dénotation par conjoint, mais qui utilisent l'entassement de plusieurs conjoints pour créer une dénotation. Un exemple assez clair de coordination non relationnelle est constitué par la paire non inversible deux ou trois (Malkiel, 1959, Lambrecht, 1984, Masini, 2006). Si comme dans l'exemple (40) un locuteur dit qu'il va résumer sa pensée en deux ou trois mots, cela ne veut pas dire qu'il le fera soit en deux, soit en trois mots, mais plutôt qu'il la résumera en quelques mots. En d'autres termes, la dénotation de deux ou trois n'est pas une fonction des dénotations de deux et trois. Deux et trois ne sont pas interprétés comme deux dénotations en relation alternative, mais comme deux éléments qui dans la construction coordinative permettent de créer une nouvelle dénotation similaire à 'quelques'.

(40) $\{$ Deux $\mid \wedge$ ou trois $\}$ mots au sujet de la révision de la Constitution malienne de 1992 (titre d'un article en ligne)

L'interprétation non relationnelle de cette construction ne résulte pas, comme on pourrait le croire, du figement de l'expression deux ou trois ${ }^{13}$. Voici d'autres exemples non figés :

(41) \$L1 mais je trouverai pas de livre sur \{ Ségolène Royal |^ou Nicolas Sarkozy \} // \$L2 "ah" non // ça $<\{$ non $\mid$ non $\} / /$ ça $<$ les livres politiques $<$ "enfin" non // (D202)

(42) Quelle plaisanterie, je dirais plutôt un chihuahua ou un caniche abandonné à la veille de départs en vacances. (article en ligne de L'Express intitulé « La bête des Vosges est-elle un loup ?»)

Dans le premier cas, Ségolène Royal ou Nicolas Sarkozy ne renvoie pas à une alternative entre ces deux seuls personnes politiques, mais à n'importe quelle personne politique comme le montre la reprise de l'interlocuteur par les livres politiques. Dans le deuxième exemple, un chihuahua ou un caniche sert à désigner n'importe quel type de petit chien.

Les coordinations non relationnelles se caractérisent par le fait que la dénotation de l'entassement n'est pas une fonction des seules dénotations des conjoints. Cela ne signifie pas que les conjoints n'aient pas de dénotation (Ségolène Royal et Nicolas Sarkozy ont évidemment une dénotation), mais que la dénotation de l'entassement ne se calcule pas de manière compositionnelle à partir des dénotations des conjoints (cf. Bonvino et al. 2009 qui parlent de coordinations non compositionnelles) ${ }^{14}$.

Nous distinguons deux grands types de coordinations non relationnelles : les coordinations hyperonymiques et les coordinations intensives.

\subsubsection{Coordinations hyperonymiques}

Nous appelons coordinations hyperonymiques les entassements dont la valeur renvoie à un hyperonyme des conjoints, c'est-à-dire à une classe qui contient la dénotation des conjoints, mais qui ne correspond pas, comme dans le cas des coordinations relationnelles, à une combinaison logique des dénotations :

(43) les gens $<$ au début $<+$ quand ils voyaient \{ un ordinateur | une souris "euh" | tout ça $\}<+$ \{ ils sav | ils savaient \} pas ce que c' était // (D005)

(44) donc $<\{$ on voit $\mid$ on le voit $\}$ sortir "euh" \{ du pain | des brioches | des trucs comme ça $\} / /$ (M023) 
(45) et petit à petit $<$ ils ont essayé d'avoir $\{$ quelque chose $\mid\}$ à Paris $\left(\left\{\mid\left\{\right.\right.\right.$ un petit studio $\left.\right|^{\wedge} \mathbf{o u}$ quelque chose comme ça $\}\}$ ) $\left\{\right.$ pour revenir voir leurs amis $\mid \wedge$ et $\wedge^{\wedge}$ puis pour y \& $\} / /($ D003)

(46) j'ai compris \{ que ça allait $\mid$ que vous étiez content $\mid$ que il y avait suffisamment $\left\{\left\{\right.\right.$ de $\left.\mid \mathbf{d}^{\prime}\right\}$ espaces verts $\mid\left\{\right.$ de $\left.\mid \mathbf{d}^{\prime}\right\}$ équipements | et caetera $\}$ \}//

Dans ces exemples, à chaque fois on construit un hyperonyme des conjoints : 'objet lié à l'informatique' dans le premier exemple, 'article de boulangerie' dans le deuxième, 'modeste pied-à-terre' dans le troisième, 'lieu ou équipement public' dans le dernier. La détermination dépend, elle, du choix du clôtureur et du caractère additif ou alternatif de la coordination : des ordinateurs, des souris et des trucs comme ça (coordination additive de noms comptables au pluriel avec l'extenseur truc comme ça) s'interprètera comme 'toute sorte d'objets lié à l'informatique', tandis que un ordinateur, une souris ou un truc comme ça (coordination alternative de noms comptables au singulier avec l'extenseur truc comme ça) s'interprètera comme 'n'importe quel objet lié à l'informatique'. Dans le premier cas, on construit une classe d'objets, tandis que dans le deuxième cas on pointe un élément quelconque de cette classe. Dans ce dernier cas, l'entassement fonctionne comme une approximation des conjoints. Une étude plus précise des différents clotureurs et de leur contribution sémantique a été entamée, dans une étude parallèle par Traverso (à par.) ; une typologie complète reste à faire.

Les coordinations hyperonymiques sont également possibles avec des conjoints verbaux :

(47) parce que il a dit [ \{ elles corromp | elles corrompront $\}$ tous mes petits "euh" officiers de district // ] "euh" $\{$ sans $\mid$ sans $\}$ \{ me connaître $\left.\right|^{\wedge}$ ni rien du tout $\} / /(D 204)$

(48) mais "euh" "euh" $\left\{\{\{\right.$ se $\mid$ se $\mid$ se $\}$ gourer $\mid \wedge$ et $\wedge$ puis chauffer comme ça $\} \mid \wedge{ }^{\wedge}$ 'est-à-dire $\left\{\right.$ dragouiller la mère $\left.\right|^{\wedge}$ ou draguer $\left.\right|^{\wedge}$ ou faire une déclaration d'amour à la mère $\left.\}\right\}<$ non // (D207)

Nous avons vu plusieurs exemples de coordinations hyperonymiques sans clôtureur, au début de cette section comme dans l'exemple précédent. On utilise dans ce cas des cohyponymes pour construire leur hyperonyme commun ${ }^{15}$. Un procédé plus " lexicalisé » consiste à utiliser des antonymes ou des cohyponymes suffisamment opposés pour créer un effet de parcours de l'ensemble de la classe. Il en résulte un effet de quantification universelle de la classe dénotée. Dans la construction jour et nuit, par exemple, la conjonction de deux pôles opposés désignant le domaine du temps, sert à quantifier universellement ce domaine : la construction peut donc être paraphrasée par tout le temps. Le même procédé est à l'œuvre dans la construction petits et grands, paraphrasable par tout le monde. Ce procédé, assez commun à l'écrit, se rencontre dans les échantillons de notre corpus caractérisés par un registre plus soutenu.

(49) Nous avons vu qu'il y avait accord pour la guerre entre les hommes d'État et les révolutionnaires, les constitutionnels et les Girondins, les aristocrates et les Jacobins. La guerre était, pour tous, un appel au destin. (Alphonse de Lamartine, Histoire des Girondins)

(50) $\{$ à chacune $\mid \wedge$ et à chacun d'entre vous $\}<+\left\{\right.$ Françaises $\left.\right|^{\wedge}$ et Français $\}$ de métropole | d'outre-mer | de l'étranger \} <+ je souhaite très chaleureusement \{ une bonne $\mid \wedge$ et une heureuse \} année deux mille // (M204)

(51) En revanche, les adultes comme les enfants, les garçons comme les filles, peuvent s'identifier au film. (article en ligne, site Quartier sans cibles) 


\subsubsection{Coordination intensive}

Nous avons identifié un autre type de coordination non relationnelle, caractérisée, cette fois-ci, par la coordination (potentiellement conjonctive) ${ }^{16}$ d'expressions identiques : il s'agit de la coordination intensive. La coordination intensive a une fonction générale d'intensification du sens exprimé par le conjoint répété. Cette fonction d'intensification se précise selon la catégorie de l'expression. Par exemple, la réitération de conjoints nominaux intensifie la quantité ('plein d'exercices', 'plusieurs dizaines d'années') :

(52) $\{$ le $\mid$ la $\mid$ le $\mid$ le $\mid$ la $\}$ grosse recette de Sarah "tu vois" < c'était de de faire $\{$ des exercices $\mid$ des exercices $\mid$ des exercices $\}$ par exemple "tu vois" pour un point de grammaire // (Valibel)

(53) mais mais mais mais $\{$ les lois sociales $\mid$ le droit de grève $\mid \wedge$ et tout ça $\}<\{$ ça s'est fait $\mid$ ça s'est fait \} sur \{ des dizaines | ^et des dizaines \} d'années // ça s'est fait sur presque "enfin" $\{$ cinquante $\mid$ cent $\}$ ans $/ /$ (Valibel)

La réitération de conjoints verbaux peut servir à intensifier la durée ou la fréquence de l'action et à marquer l'aspect continuatif ou itératif :

(54) On travaille, travaille et puis, geste malheureux, voilà notre mise en page qui bascule sur quelque chose de profondément laid, voilà une action qui bousille les longues heures d'acharnement... (web, blog)

(55) on pouvait pas s'empêcher à la fin de $\{$ Mort $\mid \wedge$ et transfiguration $\}$ de faire \{ résonner | résonner $\left.\right|^{\wedge}$ et résonner $\mid \wedge$ et encore $\}$ ces accords qui nous enchantaient // (D212)

La réitération de conjoints adjectivaux ou adverbiaux peut servir à former une sorte de superlatif ('pas trop facile', 'vraiment très difficile', 'vraiment tout le temps') ${ }^{17}$ :

(56) et puis "bon" "ben" "voilà" donc < ce qui fait que \{c' est | c' est $\}$ pas \{ facile | facile $\} / /(D 005)$

(57) c'est \{ très $\mid$ très $\}$ difficile à définir // (D202)

(58) c'est réexpliquer //+ mais \{ tout le temps | tout le temps | tout le temps $\} / /$ et et et alors < "euh" leur demander "tu vois" qu'ils te rappellent "euh" \{ certains points $\mid$ certaines règles $\mid$ et caetera $\}$ "quoi" // (Valibel)

La coordination intensive ajoute un élément de sens (l'intensification) au sens du conjoint et sa dénotation va donc au delà de la dénation du conjoint répété.

\section{Entassements de dicto}

Les entassements ne se limitent pas à opérer sur sur le plan de re : ils permettent d'accomplir des opérations diverses sur le plan de dicto, sur la façon dont le locuteur formule le contenu de son message et sur la façon dont il dénote les éléments du discours.

L'ensemble des entassements opérant sur le plan de dicto constitue un domaine au moins aussi riche que celui de la coordination. En général, dans les entassements de dicto, le locuteur entasse du matériel linguistique dans la même position syntaxique pour dénoter un seul et même élément La différence avec la coordination non relationnelle ${ }^{18}$ réside dans le fait que l'entassement ne contribue pas, en tant que signe linguistique, à la construction d'une dénotation complexe, mais que le locuteur l'utilise pour parcourir différentes possibilités à la recherche d'une bonne dénotation. En ce sens, l'entassement de dicto peut être vu comme un phénomène de performance, plutôt que de compétence. 
En prenant en compte la nature des éléments entassés et les effets communicatifs de l'entassement, nous distinguons trois types majeurs d'entassements de dicto : la disfluence, la reformulation, la double formulation.

\subsection{Disfluence}

La disfluence a été déjà bien décrite et analysée comme un type d'entassement par Blanche-Benveniste (1990, 1995, 1997), Blanche Benveniste et al. (1990). On parle de disfluence lorsque le locuteur piétine sur une position syntaxique afin d'ajuster sa formulation. Ce piétinement se traduit par un entassement de mots ou d'amorces de mots :

(59) et vous appartenez à ce qu'on appelle les flying doctors de la Mref $>$ qui est \{ une | une \} association $\{$ des médecins $\mid \wedge$ et $\{$ de $\mid$ de $\mid$ de $\}$ recherche $\}$ qui est basée $\{$ ici $\mid\{$ à $\mid$ à $\}$ Nairobi $\mid$ au Kenya \} // (D204)

(60) nous avons été chassés $\left\{\mathbf{d}^{\prime} \mathbf{A} \sim \mid \mathbf{d}^{\prime}\right.$ Alsace $\}>$ naturellement // (D204)

Ce piétinement a généralement lieu à l'initiale d'un constituant sur un élément grammatical (déterminant, préposition, auxiliaire) en attendant de trouver l'élément lexical adéquat. Ce piétinement peut conduire à la répétition de segments assez longs :

(61) alors $<\{\{\mathbf{j}$ 'a | j'avais $\}$ beaucoup | j'avais beaucoup $\}$ trop peur de m'installer ( comme ça ) seule $\{\mathbf{d} \sim \mid$ dans $\}$ la brousse // (D204)

Dans le cas des disfluences, le segment répété n'a pas d'interprétation propre, contrairement aux cas qui seront décrits dans les prochaines sections, où chaque conjoint possède une dénotation.

\subsection{Reformulation dénotative}

Un locuteur peut proposer une première formulation dénotative et y revenir à plusieurs reprises pour la remplacer par d'autres formulations. On est dans ce cas dans un procédé que nous appelons la reformulation dénotative.

(62) et j'avais \{ une circonscription | \{ un | un \} rayon d'action \} d'à peu près "euh" cent kilomètres tout autour de cet endroit // (D204)

(63) tu arrives place aux Herbes avec $\{$ une $\mid$ une $\}$ sorte $\{$ de halle | "quoi" $\{$ de $\mid$ de $\mid$ de $\}$ structure métallique $\} / /(\mathrm{M} 001)$

La reformulation a lieu généralement à l'intérieur de la même composante illocutoire. Cela ne veut pas dire que des interruptions, même très longues, ne soient pas possibles. Dans l'extrait suivant par exemple, trois UI parenthétiques viennent interrompre un entassement de reformulation \{ qui parlent pas français dont les mamans ne parlent pas français \} dont la seconde couche est très éloignée de la première, sans que pourtant il y ait eu changement de composante illocutoire.

(64) dans le vingtième $<+$ il faudrait \{ qu'il y ait \& | qu'on sépare \& | "enfin" qu'il y ait des cours de français pour les petits enfants $\{$ qui parlent pas français $\mid\}$ \}

( c'est pas compliqué quand même //

c'est pas très difficile d'apprendre le français à des petits enfants de cet âge-là //

$\{$ ça $\mid$ ça $\mid$ ça $\}$ se fait assez facilement // )

$\{$ dont les mamans ne parlent pas français \} // (D002) 
La reformulation peut se composer, surtout en dialogue, avec des procédés de négociation de la formulation et de la reférence. Nous verrons cela dans la section 5.

\subsection{Double formulation}

Les entassements de dicto qu'on vient d'examiner (disfluence et reformulation dénotative) peuvent être vus comme des entassements alternatifs, où la dénotation de la dernière couche a une relation de substitution avec les dénotations précedentes. Il existe toutefois un procédé d'entassement de dicto qu'on peut considérer comme additif. Ce procédé ne consiste pas à substituer une dénotation à une autre, mais à cumuler plusieurs dénotations pour un même élément :

(65) pour $\{$ Philippe Lemaire | ( l'avocat des parties civiles // ) $<+\{$ c'est $d \sim$ ce sont des $\}$ procédés terroristes // (M206)

La deuxième dénotation fonctionne alors comme une unité illocutoire en soi ce qu'indique notre encodage $(\ldots / /)$ qui en fait une parenthétique. Nous appelons double formulation ce procédé, très fréquent à l'oral (comme d'ailleurs à l'écrit), que nous présentons comme une extension de la construction appelée dans la littérature apposition non restrictive (Quirk et al. 1972, 1985, de Vries 2006) 19

Quirk et al. (1985 : 1301-1316) donnent une caractérisation formelle et fonctionnelle très complète des constructions d'apposition non restrictive, qu'ils caractérisent comme des relations entre syntagmes nominaux co-référents, juxtaposés, liés entre eux par une relation de coordination, dont le deuxième réalise une unité tonale indépendante. Ils montrent également que les appositions peuvent avoir des fonctions différentes : appellation, identification, désignation, reformulation intentionnelle, attribution, exemplification, particularisation.

La notion d'entassement nous a permis d'étendre cette notion en la caractérisant comme l'entassement de conjoints co-dénotationnels, pas nécessairement nominaux, pas nécessairement juxtaposés, dont le second se trouve systématiquement dans une unité illocutoire (souvent parenthétique) distincte de l'unité illocutoire qui hébèrge le premier conjoint ${ }^{20}$.

L'extension formelle de cette notion nous a amenés à élargir également la classification de ses fonctions.

Comme Quirk et al., nous distinguons les doubles formulations appellatives (les deux conjoints sont définis et le deuxième est un nom propre) :

(66) \{ le président de l'Unef | (+ Jean-Baptiste Prévot //) \} au micro de Sonia Bourane // (M206)

les doubles formulations identificatives (le premier conjoint est indéfini, le deuxième plus spécifique) ${ }^{21}$ :

(67) et à Cavalaire Pardigon où nous allions en été $<+$ il y avait \{ une famille amie à nous "euh" | (+ les Besse // ) \} et qui avait $\{$ un $\mid$ un $\}$ empire commercial "je veux dire" surtout $\{$ à $\mid$ à $\}$ Aden $/ /(\mathrm{D} 204)$

les doubles formulations désignatives (le deuxième conjoint est moins spécifique que le premier) :

(68) pour $\left\{\right.$ Philippe Lemaire $\mid\left(+l^{\prime}\right.$ avocat des parties civiles $\left.\left./ /\right)\right\}<+\{$ c'est $d \sim \mid$ ce sont des $\}$ procédés terroristes // (M206)

les doubles formulations attributives (le deuxième conjoint n'est pas équivalent au premier, mais il en désigne des propriétés) : 
(69) il y a eu en mille neuf cent dix huit sur l'ensemble de la planète on dit \{ quarante millions de décès $\mid\left(+{ }^{\wedge} c^{\prime}\right.$ est-à-dire une mortalité effroyable // $\left.)\right\} / /$

Nous avons également trouvé dans notre corpus oral un grand nombre de doubles formulations encodant une reformulation intentionnelle, souvent reliées par le joncteur c'est-à-dire et souvent reliant des conjoints non nominaux :

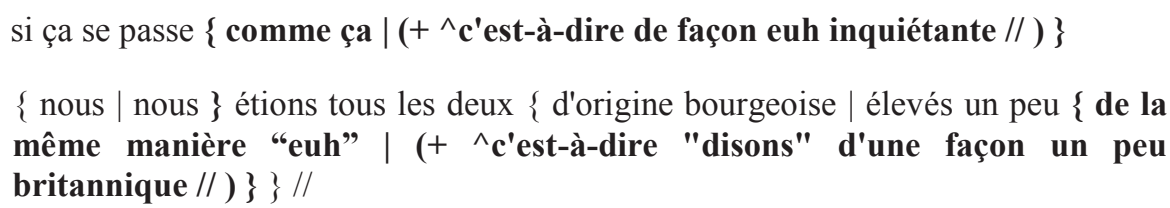

Nous avons identifié un grand nombre de doubles formulations que Quirk et al. appelleraient appositions inclusives (la dénotation du deuxième conjoint n'est pas identique à celle du premier, mais elle est incluse dans celle-ci). Celles-ci prennent soit la forme d'une particularisation (le premier conjoint est constitué par un mot général ou un pronom indéfini) ${ }^{22}$ :

(71) si je ne craignais pas d'entrer dans le jeu de certains hommes qui abusent de leur condition $<$ je dirais que vous avez donné $\{$ quelque chose de plus $\mid\}$ à la femme $/ /+\{\mid$ des armes de persuasion $\} / /(\mathrm{D} 201)$

(72) "ben" en fait < il y a \{ pas mal de choses $\mid\}$ qui rentrent en compte $/ /+\{$ déjà "euh" l'ambiance du magasin ... $\} / /$ (Olive, GARS)

(73) et j'ai trouvé \{ cet endroit | (+ Olkaloo // ) \} où ils avaient besoin d'un médecin // (D204)

soit la forme d'une exemplification (le deuxième conjoint est constitué par un entassement de cohyponymes du premier conjoint) :

(74) et j'avais absolument envie d'aller dans \{ la corne de l'Afrique $\mid\} / /+\left\{\mid\left\{\left\{\right.\right.\right.$ la $\left.\mid \mathbf{l}^{\prime}\right\}$ Éthiopie $\mid$ la

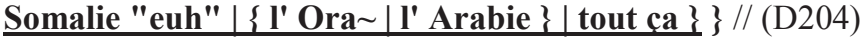

(75) "euh" et sinon < les spécialités \{ les $m \sim \mid$ un \{ peu moins ( je sais pas si c'est ça qui vous intéresse // )| petit peu moins \} $\}$ prises $<$ "bah" \{ c'est les | c'est \{ les \} spécialités à risques |\} $/ /+\{\mid$ la gynéco obstétrique ( par exemple) $\mid$ la cancérologie $\}\} / /$ (D006)

Il est à remarquer que les couches des doubles formulations inclusives sont facilement discontinues.

Parmi les doubles formulations inclusives, nous incluons aussi des constructions qui ne pourraient pas être répertoriées parmi les appositions : les questions-réponses (partielles, c'est-à-dire avec un pronom interrogatif). Malgré leur distribution sur deux UI différentes dotées chacune de sa propre force illocutoire (l'une est une question et l'autre la réponse), ces structures remplissent toutes les conditions pour être considérées comme des doubles formulations inclusives : le pronom interrogatif et l'élément utilisé en réponse occupent la même place structurale, ils sont co-dénotationnels et la dénotation du deuxième conjoint est incluse dans la dénotation du premier conjoint :

(76) \$L1 et il faut compter \{ combien de temps après $\mid\} / /$

\$L2 très rapidement "hein" // \{| \{ quinze jours | quinze jours maximum \} \} // (D009)

(77) "euh" \{ pourquoi |\} j'ai fait du journalisme //+ \{| parce que "euh" ça se passait tout de suite après la guerre $\} / /(\mathrm{D} 201)$

Notons qu'il n'y a pas lieu de parler de réponse elliptique. Comme pour l'épexégèse, le traitement de la construction comme une seule UR, même s'il y a deux UI, évite le recours à l'ellipse. 


\subsection{La négociation de la formulation}

Les locuteurs peuvent négocier entre eux la construction du discours aussi bien que l'ensemble des formulations introduites dans le discours. Comme certaines études interactionnistes (Jefferson, 1991, Selting, 2007) l'ont montré, l'entassement constitue une structure privilégiée pour ce genre d'opération, qui intéresse tout particulièrement les dialogues, mais aussi les monologues lorsqu'ils relèvent de la polyphonie.

Nous avons relevé quatre opérations de négociation opérant dans les entassements dans notre corpus : la demande de confirmation, la confirmation, la réfutation, la correction.

Lors d'une négociation par entassement, il y a répétition d'un segment avec prosodie appropriée. Les exemples suivants montrent une demande de confirmation (répétition de aux marseillais avec une prosodie interrogative, notée par?) et une confirmation (répétition de qui existe toujours avec une prosodie assertive) sans qu'il y ait eu pour cela demande de confirmation d'ailleurs :

(78) \$L1 ils avaient honte par rapport \{ aux Marseillais |\} //+

\$L2 \{| aux Marseillais \}?//+

\$L1 parce qu'ils parlaient pas le même provençal qu'eux // (corpus Debaisieux)

(79) \$L1 moi $<\{$ j'étais donc "euh" | j'étais $\}$ à l'école rue de Picpus //+

L2 \{ qui existe toujours $\mid\} / /+$

\$L1 \{| qui existe toujours $\}>$ absolument // (D001)

(80) \$L1 puisque finalement < ça fait "euh" \{ quarante-huit ans $\mid\}$ que vous êtes au Kenya // \$L2 $\{\mid$ quarante-huit ans $\} / /\{$ oui $\mid$ oui $\} / /(D 204)$

Il est aussi courant que la négociation par entassement se superpose à une reformulation. Dans (81) on a une demande de confirmation et une confirmation qui s'enchaînent. La demande de confirmation est une répétition (les quais), mais la confirmation comprend une reformulation (les quais du tram). Dans (82), c'est une reformulation (d'une jeune bourgeoise) qui s'accompagne d'une demande de confirmation et la confirmation est une répétition :

(81) \$L1 et à ce niveau-là <+ vous allez tourner "euh" à votre droite //

vous allez longer $\{$ les quais $\mid\} / /+$

( vous allez passer devant $\{\mathrm{u} \sim \mid$ une $\}$ banque //

à l' angle <+ il y a une banque pour vous repérer // )

\$L2 $\{\mid$ les quais |\} ?//+

\$L1 "euh" $\{$ les quais du tram $\} / /$

(82) \$L2 "eh bien" je crois que je ne me suis pas conduit d'une façon conforme à ce qu'on attend "euh" $\left\{\left\{d^{\prime}\right.\right.$ 'une jeune fille d'abord $\left.\right|^{\wedge}$ et d'une femme ensuite $\left.\} \mid\right\} / /+$

\$L1 \{| d'une jeune bourgeoise |\} ?//+

\$L2 \{| "disons" d'une jeune bourgeoise \} // (D201)

Une répétition peut aussi être une réfutation de l'élément répété. Dans les exemples suivant la réfutation est introduite par l'élément enfin ${ }^{23}$, qui semble spécialisé pour cet usage, et elle est suivie d'une correction

(83) et "euh" "bon" "ben" ça pose des problèmes \{ de maintenan | ("enfin" de maintenance "euh" //) $\mid\{\{$ de $\mid$ de $\}$ mise à jour $\mid \wedge$ et tout ça $\}$ "euh" $\} / /$ voilà // (D005)

(84) c'est la crise générale $\{\{$ des $\mid$ des $\}$ Français $\mid\} / /+\{($ "enfin" des Français//) $\mid$ (pas simplement des Français "hein"//) $\mid\{\{$ des $\mid$ de $\}$ l'humanité $\mid \wedge$ et de la lecture $\}\} / /$ (D004) 
Il est bien sûr possible d'avoir une correction sans réfutation préalable :

(85) alors < le problème "si vous voulez" \{ qui se pose "euh" à Paris | "enfin" que je vois dans le vingtième \}< c'est le problème des écoles \{ maternelles $\mid \wedge$ et primaires \}//

(86) et la seule école privée "euh" qu'il y avait dans le quartier ( moi ) avec qui je travaillais un peu quand je suis arrivée ici a fermé il y a maintenant "pff" peut-être au moins \{ douze ans "je pense" " hein" $\mid\} / /+\{\mid\{$ douze $\mid$ treize $\}$ ans $\} / /(\mathrm{D} 004)$

La correction, quand elle est dialogique, peut être suivie d'une confirmation :

(87) \$L1 et il donne $\{$ à Gaga $\mid\} / /+$

$\$ L 2\{\mid$ à $\{$ Gago $\mid\}\}>$ effectivement $/ /+$

\$L1 \{| Gago |\} "pardon" //

\$L2 \{| Gago \} \{ qui est contré | qui est contré \}//

(D203, commentaires en direct pour un match de foot)

La limite entre une reformulation pure et simple et une reformulation-correction, voire une reformulationconfirmation est parfois ténue comme le montre l'exemple suivant :

(88) \$L1 mais "euh" de plus en plus $<$ je vois des gens \{ qui sont respectueux $\mid \wedge$ et qui ramassent $\mid\{$ qui $\mid$ qui $\mid$ qui $\}$ prennent leur chien $\mid$ qui les font faire leur besoin $\{\{$ dans $\mid$ dans $\}$ le trottoir $\mid\}$ \} $/ /+\{\mid$ pas sur le trottoir ( justement ) $\} / /+$

\$L2 $\{$ dans le caniveau $\} / /\{$ oui $\mid$ oui $\} / /(\mathrm{D} 006)$

(89) une fois qu'on a goûté Paris <+ on veut plus aller à la province //+ \{ pour des études | "non" pour les études $\}>\{$ sûrement $\mid$ sûrement $\}$ " $\{$ ouais | ouais $\} " / /$ (D204)

Les négociations que nous avons présentées jusque dans notre corpus portaient essentiellement sur la formulation. Dans ces exemples, lorsqu'on reformule, c'est plus pour mieux dire les choses, que pour dire autre chose. Mais si on prend un exemple construit comme :

(90) \$L1 je vais inviter $\{\mid$ Paul $\} / /$

\$L2 \{| Pierre \} "non" //

on voit que la correction peut porter sur le de dicto (tu es sûr que celui dont tu parles s'appelle bien Paul) ou sur le de re (tu es sûr que tu ne voudrais pas inviter Pierre plutôt que Paul). La limite entre les deux n'est pas toujours facile à saisir. Dans l'exemple suivant, le locuteur \$L2 semble concéder une nouvelle valeur :

(91) \$L1 il faut compter "euh" "pff" \{ \{ l'équivalent de quarante euros | quelque chose comme ça \} $\mid\} / /$

\$L2 "ah" "oui" // "ah" je pensais plus que ça //

\$L1 "oui" // \{| \{ quarante | quarante-cinq \} \} // (D009)

Ce nous conforte dans notre choix de traiter la négociation comme une dimension orthogonale aux autres, puisque la négociation peut s'employer seule ou se superposer à une reformulation, voire une coordination.

\section{Conclusion}

L'entassement a été caractérisé comme un véritable objet syntaxique dans la linguistique française du siècle dernier. Nous avons étudié dans cet article les différentes valeurs sémantiques et fonctionnelles de cet objet. Nous pouvons synthétiser notre classification en présentant la hiérarchie complète (Figure 1). 
Certaines de ces valeurs sont très rarement étudiées, comme les coordinations non relationnelles ou certains types de double formulation, et seules la perspective adoptée et l'étude sur corpus nous ont permis de les dégager. D'ailleurs, peu d'études font le lien entre les différents phénomènes d'entassements et en particulier entre les phénomènes de coordination et de reformulation.

En situant notre étude dans un modèle distinguant phénomènes micro et macrosyntaxiques, nous avons pu identifier les propriétés formelles caractérisant les différents types d'entassement. Nous avons vu que certains types d'entassements, comme la double formulation ou la négociation sont par définition sur deux unités illocutoires distinctes et certains peuvent être discontinus.

La position des entassements par rapport à la structure macrosyntaxique de l'énoncé joue un rôle important dans la définition de la valeur de l'entassement, mais aussi la relation sémantique entre les conjoints, la nature de la jonction, l'ensemble des marqueurs (joncteurs, clôtureurs, adverbes, marqueurs discursifs) présents dans l'entassement contribuent à en déterminer la valeur.

Quand un entassement est produit avec un nombre restreint de marqueurs, il peut présenter une ambigüité de valeur et il peut arriver qu'il puisse être interprété à la fois comme une coordination ou une reformulation ou encore comme une intensification ou une confirmation. Dans la plupart des cas cette ambigüité n'est pas gênante: accepter l'une ou l'autre valeur change peu l'interprétation globale de l'énoncé. Cela prouve la contiguité fonctionnelle, outre que formelle des entassements et justifie de considérer les entassements dans leur globalité.

Il est intéressant de noter que les entassements effectuent des opérations dont la valeur est aussi celles d'éléments grammaticaux: intensification, dérivation hyperonymique, détermination, etc. Les entassements jouent notamment un rôle important à l'oral où la nécessité de maintenir un flux constant oblige le locuteur à laisser de nombreuses traces de la construction du sens, de choix dans la dénotation, etc. (Blanche-Benveniste et al., 1990).

Une typologie fine des différentes valeurs « grammaticales » des entassements prenant en compte le rôle joué par les joncteurs et les clôtureurs dans la détermination précise du signifié reste à faire. Cette analyse ne pourra pas ne pas prendre en compte la dimension prosodique, ce qui pourra être fait lorsque les annotations syntaxiques et prosodiques du corpus Rhapsodie seront distribuées conjointement.

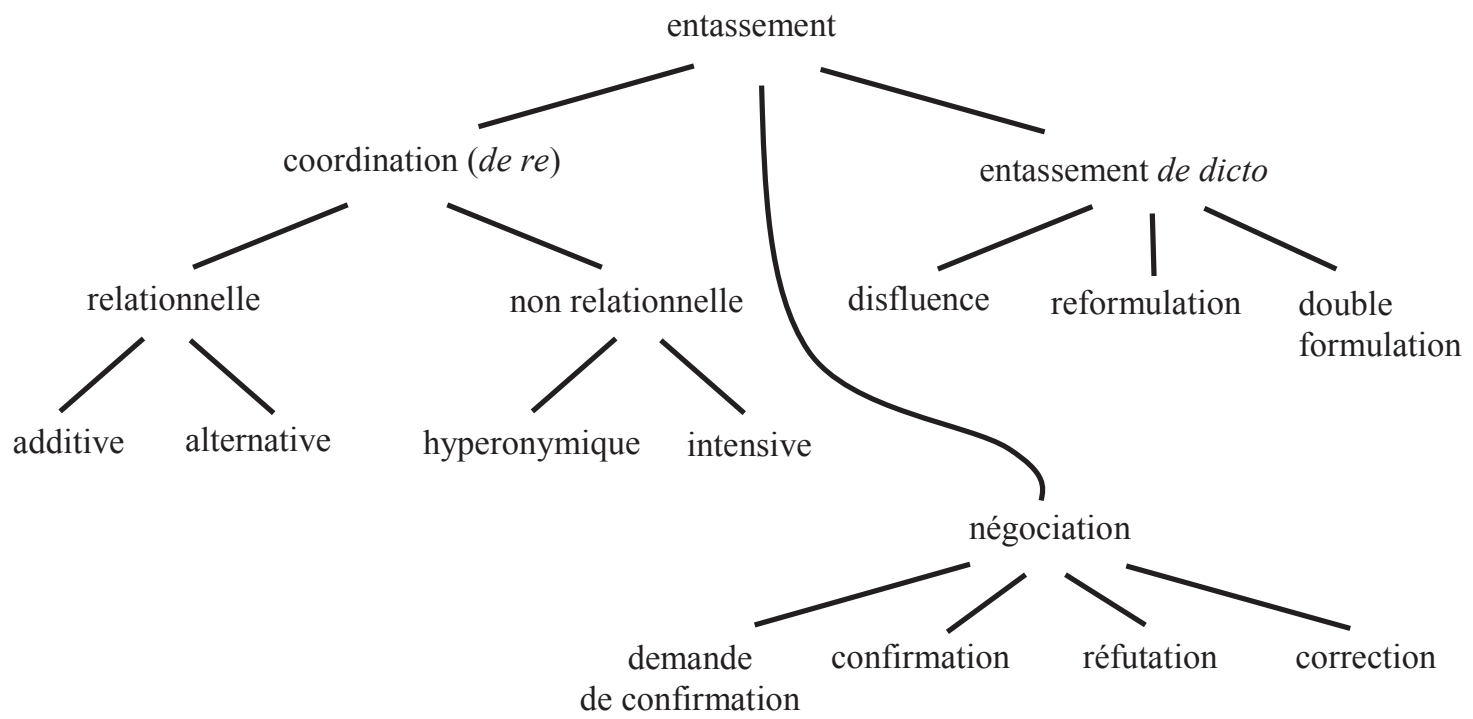

Figure 1. Hiérarchie des types d'entassements 


\section{Références}

Abeillé, A. (2005). Les syntagmes conjoints et leurs fonctions syntaxiques. Langages, 39, 42-66.

Bally, Ch. (1932). Linguistique générale et linguistique française, Berne, A. Francke.AG Verlag,

Benzitoun, Chr., Dister, A., Gerdes, K., Kahane, S. \& Marlet, R. (2009). Annoter du des textes tu te demandes si c'est syntaxique tu vois. Arena Romanistica, 4, 16-27.

Benzitoun, Ch. Dister, A., Gerdes, K., Kahane, S., Pietrandrea, P. \& Sabio F. (2010). Tu veux couper là faut dire pourquoi. Propositions pour une segmentation syntaxique du français parlé. Congrès Mondial de Linguistique Française (CMLF 2010), 2075-2090.

Bilger, M. (1989). Les réalisations en et tout (ça) à l'oral, Recherches sur le français parlé, 9, 97-109.

Bilger, M. (1999). Coordination: analyses syntaxiques et annotations. Recherches sur le français parlé, 15, 255-272.

Blanche-Benveniste, Cl. (1997). Approches de la langue parlée en français. Paris : Ophrys.

Blanche-Benveniste, Cl. (1990). Un modèle d'analyse syntaxique "en grilles" pour les productions orales. Anuario de Psicologia, 47, 11-28.

Blanche-Benveniste, Cl. (1995). Le semblamble et le dissemblable en syntaxe. Recherches sur le français parlé 13, 7-33.

Blanche-Benveniste Cl. (à par). Les beautés de l'énumération. 8 p.

Blanche-Benveniste, C.; Deulofeu, J.; Stefanini, J. \& van den Eynde, K. (1984). Pronom et syntaxe. L'approche pronominale et son application au français, Paris : SELAF

Blanche-Benveniste, Cl., Bilger M., Rouget Ch. \& van den Eynde, K. (1990). Le français parlé. Etudes grammaticales. Paris: Editions du Centre National de la Recherche Scientifique.

Bonvino, E., Masini, F. \& Pietrandrea P. (2009). List Constructions: a semantic network. Troisième Conférence Internationale de l'AFLiCo, Nanterre. Accessible à http://francescamasini.caissa.it/Presentations_files/ parigi_draft.pdf.

Deulofeu J., Duffort L., Gerdes K, Kahane, S. \& Pietrandrea, P. (2010). Depends on What the French Say Spoken Corpus Annotation With and Beyond Syntactic Functions. Proceedings of the Fourth Linguistic Annotation Workshop (LAWIV), Uppsala : ACL.

van den Eynde, K. \& Blanche-Benveniste, Cl. (1978). Syntaxe et mécanismes descriptifs: présentation de l'approche pronominale, Cahiers de Lexicologie, 32, 3-27

Gerdes K. \& Kahane S. (2009). Speaking In Piles: Paradigmatic Annotation Of French Spoken Corpus. Proceedings Of The Fifth Corpus Linguistics Conference, Liverpool, 15 p.

Guénot, M.-L. (2006). La coordination considérée comme un entassement paradigmatique: description, formalisation et intégration. In Mertens, P., C. Fairon, A. Dister \& P. Watrin (éds), Cahiers du Cental, 2:1, Verbum ex machina, Actes de la $13^{\text {ème }}$ Conférence sur le Traitement Automatique des Langues, Leuven, Belgique, 10-13 April 2006, 1, 178-187.

Jefferson, G. (1991), List construction as a task and resource, in Psathas G. (ed.), Interactional competence, New York, Irvington Publishers, 63-92.

Kahane, S. (à paraître). De l'analyse en grille à la modélisation des entassements. In Caddeo, S, Roubaud, M.N., Rouquier, M., Sabio, F. (éds), Hommage à Claire Blanche-Benveniste, Presses de l'université de Provence. 
Kahane, S. \& Pietrandrea, P. (2012). Les parenthétiques comme « Unités Illocutoires Associées » Une perspective macrosyntaxique. Linx, 61 49-70.

Lambrecht, K. (1984). Formulaicity, frame semantics, and pragmatics in German binomial expressions. Language, 60 (4), 753-796.

Malkiel, Y. (1959). Studies in irreversible binomials. Lingua, 8, 113-160.

Masini, F. (2006). Binomial constructions: inheritance, specification and subregularities. Lingue e Linguaggio, V (2), 207-232.

Mauri, C. (2008). Coordination relations in the languages of Europe and beyond. Berlin \& New York: Mouton de Gruyter.

Ndiaye M. (1989). L'analyse syntaxique par joncteurs de liste. Thèse de Doctorat, Université d'Aix-Marseille.

Nølke H. (1983). Les adverbes paradigmatisants : fonction et analyse. Copenhague, Akademisk Forlag.

Overstreet M. (2005). And stuff und so: Investigating Pragmatics Expressions in English and German. Journal of Pragmatics 37, 1845-1864.

Rousseau, A. (2001). Un cadre de logique naturelle pour la description des relations de coordination. In Rousseau, A. (éd.), La sémantique des relations. Lille 3, CEGES, 195-212.

Selting, M. (2007). Lists as embedded structures and the prosody of list construction as an interactional resource. Journal of Pragmatics, 39, 483-526.

Tesnière L. (1959). Éléments de syntaxe structurale. Paris, Klincksieck.

Traverso V. (à paraître). Listes et mise en place de classes d'objets dans les échanges ordinaires. In Dufiet, J.P. (éd.), Synonymie au-delà du lexique, Udine : Forum.

\footnotetext{
${ }^{1}$ Nos conventions d'annotation des exemples vont être présentées dans la section 2.
}

${ }^{2}$ Une version non définitive du corpus annoté en macrosyntaxe est consultable à http://rhapsodie.ilpga.fr/ wiki/Corpus.

${ }^{3}$ La notion de rection que nous utilisons ici a été élaborée dans plusieurs cadres théoriques, notamment dans la grammaire de dépendance à la suite de Tesnière (1959) et dans l'approche pronominale à la suite des travaux de Karl van den Eynde et Claire Blanche Benveniste (van den Eynde \& Blanche-Benveniste, 1978 ; Blanche-Benveniste et al., 1984). Les détails des différences entre ces conceptions de la dépendance et d'autres ne sont pas pertinents ici.

${ }^{4}$ Les joncteurs sont plus ou moins les conjonctions de coordination. Nous adoptons ici, à la suite de BlancheBenveniste et de Ndiaye (1989), une variante du terme jonctif utilisé par Tesnière (1959). Voir aussi le terme marqueur d'entassement (pile marker) de Gerdes \& Kahane (2009).

${ }^{5}$ Le lexème et caetera fonctionne ainsi comme l'amalgame d'une joncteur et d'un extenseur, ce qu'il est à l'origine. Cela explique qu'il ne puisse pas, comme les joncteurs, apparaître en dehors d'un entassement.

${ }^{6}$ Dans cet exemple aussi, plusieurs couches ne sont pas syntaxiquement saturées, ce que nous indiquons par le symbole \&.

${ }^{7}$ C'est cette propriété qui permet de décrire la sémantique des joncteurs et et ou dans les coordinations de ce type par des tables de vérité, lorsque le sens (confondu ici avec la dénotation) d'une proposition est défini comme l'ensemble des situations où cette proposition est vraie. 


\begin{abstract}
${ }^{8}$ Le joncteur $n i$ s'utilise dans la portée d'un élément négatif. Notre deuxième exemple est de ce point de vue assez intéressant, puisque stricto sensu ni n'est pas dans la portée d'une négation (*les formations ne sont pas faites pour les gens illettrés ni déscolarisés) et il y a donc un phénomène de contamination au joncteur de la négation que contient les conjoints.
\end{abstract}

${ }^{9}$ Le lexème puis remplit bien la condition définitoire des jonctifs $(*$ ils mettaient leurs enfants puis dans le privé). Mais il peut être précédé de et (ils mettaient leurs enfants d'abord dans le public et puis dans le privé), ce qui en fait un joncteur plus faible que les autres.

${ }^{10}$ De nombreux linguistes (voir encore Mauri (2008) récemment) considèrent les coordinations adversatives comme un troisième cas à côté des coordinations additives et alternatives. Le caractère adversatif de mais en fait un connecteur fondamentalement binaire, à la différence de et, mais il n'en est pas moins parfaitement additif.

${ }^{11}$ Tesnière (1959 : 352) considère que même dans un emploi comparatif tel que Alfred aime Bernard comme un frère), comme assure la jonction entre un frère et soit Alfred, soit Bernard. Même si comme est ici régi par le verbe aime (voir le test qui suit pour distinguer les emplois non régis), il y a effectivement une relation paradigmatique qui se crée entre un frère et soit Alfred (Alfred aime Bernard comme un frère aime Bernard), soit Bernard (Alfred aime Bernard comme il aime un frère).

${ }^{12}$ Le test que nous utilisons ici pour distinguer jonction et rection a été déjà utilisé, notamment pour contraster car et parce que (Abeillé 2005): seul le deuxième accepte la coordination et marque donc une subordination. Un autre test classique est celui de l'accord pluriel des coordinations : le lexème comme qui permet les deux accords (Marie comme Pierre viendra/viendront) se situe à la frontière entre coordination et rection.

${ }^{13}$ Même si cette expression est bien figée comme d'autres coordinations non relationnelles : plus ou moins, jour et nuit, petits et grands, etc.

14 Il est clair que, dans le cadre d'une sémantique dénotative, ces coordinations sont non compositionnelles. Néanmoins, si l'on s'en tient au sens linguistique (les signifiés des signes et non leur dénotation), on peut attribuer aux coordinations non relationnelles vues en tant que constructions un signifié et calculer le sens de l'entassement en composant selon des schémas de composition réguliers ce signifié avec celui des conjoints.

${ }^{15}$ En italien par exemple, la classe des animaux domestiques est souvent désignée par cani, gatti e canarini 'chiens, chats et canaris'. On peut faire la même chose en français, bien que le procédé soit moins lexicalisé.

${ }^{16}$ Nous parlons de conjonction potentielle parce que dans la plupart des occurrences les entassements à valeur d'intensification ne présentent pas de joncteurs. Néanmoins, il est toujours possible d'ajouter à une coordination intensive une dernier conjoint précédé par et encore.

${ }^{17}$ On notera tout de même le figement de pas facile facile ou de très très.

${ }^{18}$ La contiguïté des deux opérations, entassements de re et entassements de dicto a été plusieurs fois mise en évidence par Claire Blanche-Benveniste (1990, à par.) qui a montré comment sous certains conditions on peut avoir de cas de recouvrement/fusion entre entassements de re et de dicto, l'entassement se prêtant à l'une ou l'autre opération sans que l'interprétation change pour autant. Dès qu'on entasse des quasi-synonymes sans joncteurs, comme dans les exemples suivants, il est difficile de faire la différence entre une reformulation et une coordination hyperonymique d'approximation :

c'est une conférence de philosophie // mais c'est aussi \{ une conférence $\{$ de $\mid$ d' $\}$ histoire de l'art | une conférence d'esthétique \} // (M202)

et puis après <+ "ben" j' ai travaillé sur \{ les micro-processeurs | l' informatique \} // (D005)

La prosodie peut néanmoins permettre de trancher. Ainsi le dernier exemple apparaît clairement comme une coordination à l'écoute (prosodie de liste avec répétition du même schéma intonatif).

${ }^{19}$ Il est important de préciser que toutes le appositions prédicatives ne constituent pas des entassements. Dans une apposition "sans article" (Quirk et al., 1985: 1313) comme Philippe Lemaire, avocat des parties civiles, le deuxième 
conjoint ne remplit pas toutes les conditions morphologiques pour commuter avec le premier : syntaxiquement, ce type d'apposition ne constitue pas un entassement. Nous considérons également que les appositions restrictives comme Madame Ségolène Royal ne constituent pas des entassements. Les deux éléments des appositions restrictives sont liés par une relation de modification restrictive. Ils entrent dans une relation de dépendance syntaxique et non pas d'entassement (cf. Quirk et al., 1985 pour une position similaire).

${ }^{20}$ Le fait que le deuxième conjoint d'une double formulation réalise une unité illocutoire indépendante est prouvé par le fait qu'il peut être modifié par un adverbial d'énonciation portant sur l'illocution, tel que heureusement, franchement, bref.

${ }^{21}$ On notera que dans cet exemple, la parenthètique entassée (+ les Besse // ) vient s'insérer dans une coordination une famille \{amie à nous |^et qui avait un empire commercial ... \}. Pour l'encodage de ces entrelacements dans Rhapsodie, cf. Benzitoun et al. (2009). Voici un autre exemple d'entrelacement :

et comme j' étais le seul informaticien < "euh" "ben" \{ça $\left.\mid \mathrm{c}^{\prime}\right\}$ est devenu $\{$ assez dur $\mid\} / /$ \{ puisque maint ( $\{\mid$ "enfin" dur relativement $\} / /) \mid$ puisque maintenant $\}<$ je suis responsable \& // (D005)

${ }^{22}$ Ces constructions ont été nommé effet deux-point par l'équipe du GARS (Blanche-Benveniste et al 1990), en raison de leur transcription par un deux-point à l'écrit (correspondant dans notre annotation à $/ /+$, marquant une fin d'UI qui n'est pas une fin d'UR) :

Je dirais que vous avez donné quelque chose de plus à la femme : des armes de persuasions.

${ }^{23}$ Le statut exact de enfin a été l'objet de nombreux débats au sein de l'équipe de Rhapsodie. Il est noté ici comme un marqueur de discours, mais il n'est pas exclu qu'il soit un joncteur. 